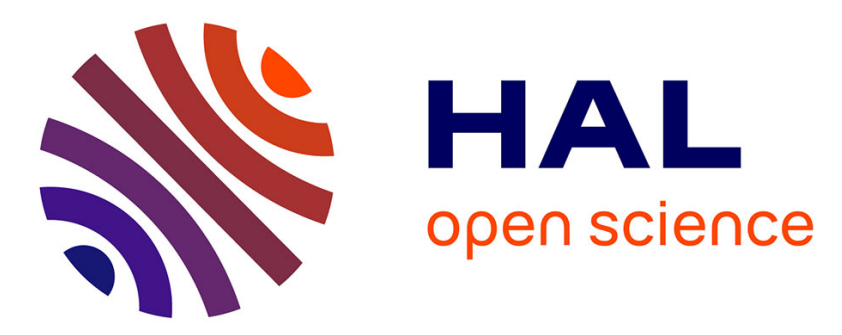

\title{
Some of the Magnetic Properties of Polycrystalline Soft Ferrites: Origins and Developments of a Model for the Description of the Quasistatic Magnetization
}

\author{
M. Le Floc'H, A. Konn
}

\section{- To cite this version:}

M. Le Floc'H, A. Konn. Some of the Magnetic Properties of Polycrystalline Soft Ferrites: Origins and Developments of a Model for the Description of the Quasistatic Magnetization. Journal de Physique IV Proceedings, 1997, 07 (C1), pp.C1-187-C1-190. 10.1051/jp4:1997169 . jpa-00255116

HAL Id: jpa-00255116

https://hal.science/jpa-00255116

Submitted on 1 Jan 1997

HAL is a multi-disciplinary open access archive for the deposit and dissemination of scientific research documents, whether they are published or not. The documents may come from teaching and research institutions in France or abroad, or from public or private research centers.
L'archive ouverte pluridisciplinaire HAL, est destinée au dépôt et à la diffusion de documents scientifiques de niveau recherche, publiés ou non, émanant des établissements d'enseignement et de recherche français ou étrangers, des laboratoires publics ou privés. 


\title{
Some of the Magnetic Properties of Polycrystalline Soft Ferrites: Origins and Developments of a Model for the Description of the Quasistatic Magnetization
}

\author{
M. Le Floc'h and A.M. Konn
}

\author{
Laboratoire d'Électronique et des Systèmes de Télécommunications, URA 1329, UFR Sciences, BP. 809, \\ 6 avenue Le Gorgeu, 29285 Brest cedex, France
}

\begin{abstract}
Since the first laws discovered by Lord Rayleigh in 1887 , the literature has given a spectacular number of works in the magnetisation modeling field. The authors remark that most of these works are devoted to soft magnetic metals with intent to improve the knowledge of the dynamic power losses (anomalous losses), and that only a few models have really been developed on true physical considerations. In the soft ferrites area, Globus has proposed a model of magnetisation based on the motions (reversible bulgings and irreversible movements of translation) of a single $180^{\circ}$ domain wall contained in an ideal spherical grain. The authors discuss the main assumptions of this model with regard to their own practical experience of soft ferrites (actions of external anisotropies), and also to some particular results published by other workers. In the last part of this paper, a new progress on the Globus model is presented which concerns the hysteresis simulation. The result is tested on actual hysteresis loops of different shapes.
\end{abstract}

Polycrystalline soft magnetic materials have a magnetisation which generally depends on both domains rotations and domain wall motions. If the latter contributes the major part of the permeability, its sensitivity to the presence of irregularities (voids, inclusions, fluctuations of compositions, internal strainings, crystal boundaries) may easily make materials of the same intrinsic properties quite different in their macroscopic responses. An experimental proof of the basic role played by the microstructure on the magnetisation processes was brougth in 1950 by Rado et al.[1] who observed in bulk Mg-ferrites two peaks of frequency losses (a low frequency peak due to the domain wall resonance, and a high frequency one due to the rotation resonance), but only the rotational peak when ferrites were powdered then dispersed in paraffin. Similar observations were also reported by Brown and Gravel[2] on very porous Ni-ferrites, confirming that only the effects of rotations were visible in spite of a material densification sufficient to allow domain walls to exist. So, in such microstructures disturbed by distributed air-gaps (cases of non uniform saturation magnetisation[3]), multidomain structures are present but the domain walls being heavily hindered, finally must have a rather low contribution to the total magnetisation. Insofar as magnetisation modelings are considered, it seems thus logical to examin how parameters of microstructure could be taken into account. After DeBlois[4]: ".. in an ideally homogeneous magnetic material, a contained domain wall could be moved infinitely slowly with no irreversible loss of energy, namely with no hysteresis. Its motion would be entirely reversible in the thermodynamic sense ..". Actually, bulk materials, even carefully densified to avoid the air-gaps mentionned above, nevertheless show a certain amount of inhomogeneities which cause irreversible energy losses for domain walls as soon as they move over appreciable distances. These defects are generally considered in theories [5,6] as pinning centres which force the domain walls to move reversibly until the energy becomes sufficient to break them away from the pinning sites. This phenomenological aspect has naturally led to a representation of the specimen's potential energy as an aleatory series of more or less pronounced " peaks and valleys". It gave the possibilities of undertaking computations on the hysteresis modeling. As an example was the demonstration of Néel[7] concerning the experimental laws discovered by Lord Rayleigh in 1887[8]. Later, the literature produced a spectacular number of models of magnetisation and magnetic hysteresis, however essentially concerned by metals and the crucial problem of the frequency power losses (eddy currents and anomalous losses in conducting media[9-11]). One notice surprisingly that, except a few number of models based on physical considerations as the works of Jiles and Atherton on metals[12,13], or those of Knowles or Dionne on ferrites[14,15], or the various developments of the famous model of Preisach[16-20], a great number of studies put forwards purely mathematical solutions [21-28]. As a matter of fact, approximation methods by power series, Fourier series or hyperbolic forms are able to follow precisely any magnetisation curve and are also often more convenient to use than refined physical models[29-32].

Magnetic metals are different from ferrites insofar as the grain sizes are generally much larger so that domain wall motions are rather controlled by distances between intragranular defects than by crystal boundaries as in high quality ferrites[33,34]. ICF7 is a congress on ferrites, so we shall only speak about ferrimagnetic materials and more specially on a physical model of magnetisation that Globus initiated in 1963[35] then detailed with many experimental supports at ICF2 in 1976[36].

Let us imagin a " perfect " polycrystalline material of magnetically soft substance formed of defect-free single crystals separated by thin boundaries. Suppose the grains as divided in antisymmetric bi-domains by $180^{\circ}$ domain walls moreover ideally coupled to warrant the magnetic flux continuity throughout the sample. Microstructure on one hand, and the hysteresis property on the other, are 
then introduced respectively by a mean grain size parameter and a domain wall-to-grain boundary coupling parameter. All grains in the material have similar behaviours so that they can be replaced by a single one (see also Knowles [14]), whose shape has initially been chosen as spherical. In the demagnetisation state, the $180^{\circ}$ domain wall is in the diametral position. Under the action of a field, the domain wall bulges reversibly until a threshold field (Hcr) is reached involving an irreversible movement of translation in grain. The permanent solid friction of the domain wall against the grain boundary and its surface variations during the displacement are both at the origin of hysteretical losses.

\section{ON THE GRAIN SIZE EFFECT}

The effect of grain size on the permeability of soft ferrites has been clearly reported by several authors, either through linear functions (Figs. (1-a) and (1-b)[33-38]) or through more or less inflected curves [39,40] suggesting, as is usual in metals[41], an upper limit of grain size beyond which domain walls are sized by distances between intragranular defects rather than by the grain diameter itself (note however that a linear relationship of Globus model-type has been found also in metals [427). By a study on neutron depolarisation, Van der Zaag et al.[43] have shown in $\mathrm{Mn}-\mathrm{Zn}$ ferrites that single-domain grain structures changed in antisymmetric bi-domain grains beyond dimensions of 3 or 4 microns (confirmed by theoretical computations based on the assumption of a domain wall located in the equatorial plane of the sphere). Following the same route as Visser et al.[44], we have evaluated the conditions of existence of a single domain structure in $\mathrm{YIG}, \mathrm{Ni}$ - and $\mathrm{Ni}-\mathrm{Zn}$ ferrites. Critical sizes $\mathrm{D}_{\mathrm{C}}$ respectively equal to $0.2-0.1-0.08 \mu \mathrm{m}$ have been found. So, except $\mathrm{Mn}-\mathrm{Zn}$ ferrites which would magnetise by spin rotations up to grain sizes of a few microns [43-45], we conclude that many other micronic grain ferrites have a magnetisation primarily controlled by domain wall motions. In these cases the model of Globus can be fully applied. Fig. (1-c) shows that the grain size effect is valuable over the whole magnetisation scale (at the same value of the remanent state, hysteresis loops of samples of different mean grain sizes can be reduced to a single loop by only acting on the grain size parameter [36]). Obviously, any intragranular defect increasing tends to reduce the influence of the grain boundary, so that in these cases an average domain wall size parameter will be better to interpret the magnetization processes [46].

\section{ON THE SPONTANEOUS DOMAIN WALL ARRANGEMENT}

Speaking about his model, Knowles said in 1959: “.the grain is typical of all grains in the material, each grain has one (or two $180^{\circ}$ domain walls) which do not in general cross the grain boundaries, but may do so if the orientations of adjacent grains are favourable."[14]. Indeed, it is possible to regard the bulk material as an assembly of independent bi-domain grains oriented at random. The same author continues as follows: ". .the magnetisation of a grain may alter independently of that of adjacent grains, the effects of free poles on the grain boundaries will be neglected. ". Actually, these free poles can simply be avoided by imaging (as the author says above) the $180^{\circ}$ domain walls in adjacent grains have the possibility to align (at least locally) along a common direction chosen on a flux continuity criterion (effect of spontaneous alignment). This is quite plausible in soft ferrites since grains have several easy axes and a rather low anisotropy field. From an ideal point of view, ring-shaped soft ferrites with (theoretically) defect-free grains would be then able to show a specifical domain wall arrangement following the ring circonferences (Fig.2-a)[36]. Many investigations have been devoted to evaluate the probability of this so-called " magnetic ring " concept. All of them led to satisfactory supports[47-52], the most convincing examples being presented in Figs. (2-b), (2-c) and (2-d): (i) - Since $\lambda_{\mathrm{s}}$ in YIG is negative, a mechanical compression $(\sigma)$ causes domains to rotate in its direction. In Fig. (2-b), $(\sigma)$ is applied in the measurement direction, namely along the " presumed favourable " direction that domains would take spontaneously (ring circumferences). The conclusion always was that, in defect-free grain samples (noted T), the magnetoelastic effect was negligible with regard to the increases of susceptibility systematically observed in materials (noted D) containing intragranular microporosity[49]. (ii) - Reminding that no precession arises in r.f. field when is applied parallel to the direction of spins, inversely, any absence of a resonance peak is a serious argument in consideration of the probability of this parallelism. In Fig. (2-c), the r.f. field is applied along the circumferences (TEM mode in coaxial line), so that we conclude that samples (T) obey the " magnetic ring " concept, but not the samples (D) (presence of a resonance peak[52]). (iii) - Assuming finally that samples (T) show a spontaneous magnetisation parallel to the ring circumferences, the axis of revolution of these samples should behave as a hard direction of magnetisation. Fig. (2-d) shows effectively that the susceptibility is rigorously unchanged until a threshold field $\left(\mathrm{H}_{B}\right)$ is reached and beyond which the susceptibility magnitude falls down quickly. $\mathrm{H}_{B}$ has been identified as an anisotropy field responsible for the toroidal alignment[53]. Materials (D), on the contrary, show a rather smooth decreasing associated to an usual hysteretical behaviour[51].

\section{APPLICATION TO THE MAGNETISATION MODELING}

Fig. 3-a shows the different stages of magnetisation attached to some particular positions of the $180^{\circ}$ domain wall in the spherical grain model of Globus. In the demagnetised state the domain wall is flat and in diametral position. Under weak fields, it bulges reversibly while above Hcr, the displacements become irreversible. Being continuously pinned to the grain boundary, the domain wall keeps the last position as the field applied is removed (remanent state). Is the field is increased now negatively, the domain wall bulges in the opposite sense, then breaks off for exactly the same magnitude as the positive field previously applied just before the removing. There is thus no intermediate balance state up to the symmetrical position in grain[36]. Cancelling the negative freld applied, leads then to the opposite remanent state. Let us now consider the quantitative aspects of the problem : under the magnetic pressure, the domain wall moves towards a new balance position which depends on both the losses due to the pinning and depinning effects at the grain boundary (characterized by a coefficient $f:$ strength by length unit of the sphere circumference) and those due to the changes in the domain wall surface (characterized by the wall energy density $\gamma$ ) during its displacement. These assumptions led 
Guyot in $1975[54]$ to propose an expression for the hysteresis losses (E) in connection with the grain diameter (D) and the reduced remanent magnetisation $\left(m_{R}=M_{R} / M_{S}\right)$. Starting from this model of energy, we gave later a new parametric formulation for the first magnetisation curve which was successfully confirmed by experimental studies devoted to the approach to saturation[50]. However, if the initial form of the Globus model [Fig. (3-a)] is able to qualitatively describe the character of irreversibility, it does not coincide energetically (the area of the loop is too large) with the model of Guyot, and consequently does not agree any longer with our new parametric formulation of the first magnetisation. The discrepancy comes from the fact that the model supposes that the reverse depinning field has always the same (absolute) value as the direct field corresponding to the loop tips, leading so to unrealistic vertical sides. Actually, many experiments have shown that the loop sides have the same properties as the first magnetisation curve. Therefore, we have proposed to extend the parametric equations of the first magnetisation curve to the description of the loop sides. Fig. (3-b) gives, in medium fields, the transformation of the loop shape: the loop sides depend now on the same parameters as the first magnetisation curve (agreement with experimental results), and the loop area is exactly given by the energy model of Guyot. Fig. (3-c) is the results of further improvements (introduction of additional parameters as magnetic hardness and demagnetising effects) which has allowed the final model to be practically usable over the whole field range[55] (note the similarity with the experimental loops shown at the beginning of this text [Fig. (1-c)]). This new presentation of the Globus model is well adapted to simulation works in the field of insulating polycrystalline soft ferrites. The further development would consist now to introduce the eddy current effects to have an extension to conducting materials.

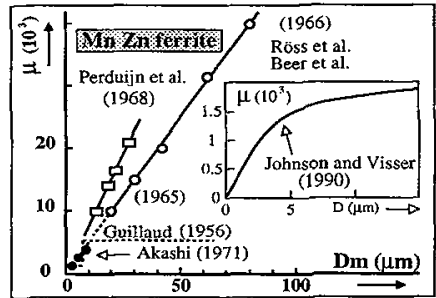

a)

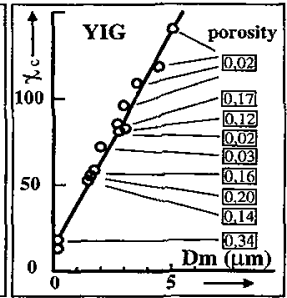

b)

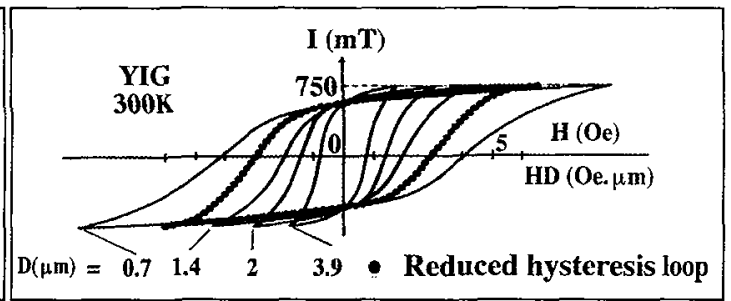

c)

Fig. $1:(a, b)$ - Effect of grain size on the permeability of polycrystalline soft ferrites [33-40]. The inflected curves probably show an excess of pinning centres in grains leading to consider an average domain wall size lower than the mean grain size. (c) - Reduction of an hysteresis loop family by only acting on the grain size parameter [36].

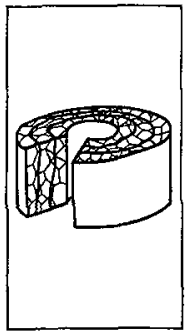

a)

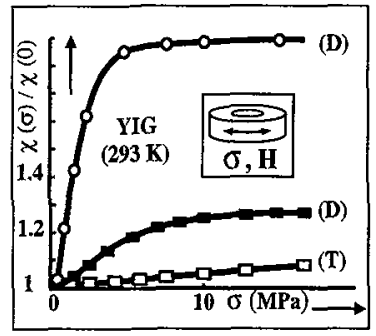

b)

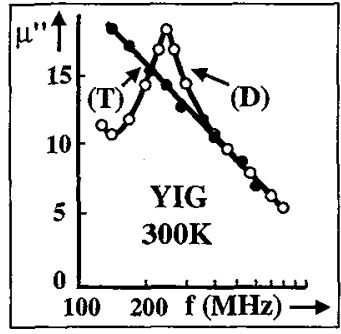

c)

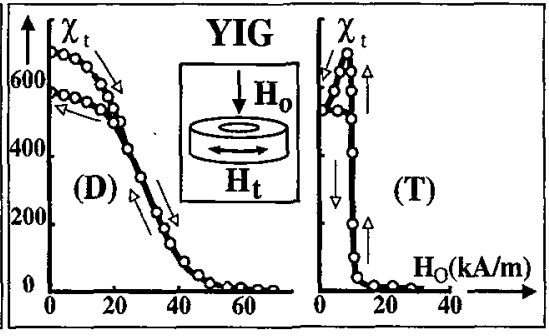

d)

Fig. 2 : (a) - Assumption of a ring-arrangement of the $180^{\circ}$ domain walls in ideal toroidal soft ferrites. (b, c, d) - Experimental confirmations in three quite different domains of investigations of the previous hypothesis (valuable only in a certain class of samples noted T [47,51-53]).

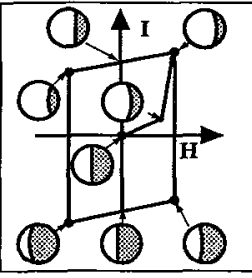

a)

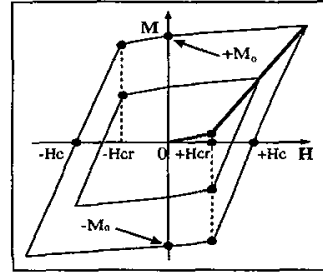

b)

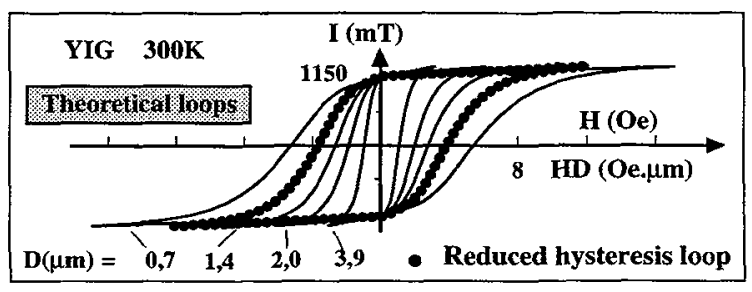

c)

Fig. 3 : Recent progress in the Globus model showing some stages of its transformation : (a) - Initial form [35,36]. (b) - Introduction of the hypothesis that the loop sides have the same properties as the first magnetization curve (example limited to moderate magnetisations). (c) - Introduction of additional parameters connected to the high magnetisation region (magnetic hardness and demagnetizing field parameters). This family of theoretical loops looks strikingly like the one of Fig. 1 (c). 


\section{References}

[1] Rado G.T., Wrigth R.W., Emerson W.H., Phys. Rev. 80 (1950) 273

[2] Brown F., Gravel Ch. L., Phys. Rev. 97 (1955) 55

[3] Schömann E., J. Appl. Phys. 38 (13) (1967) 5027-5034

[4] DeBlois R.W., J. Appl. Phys. 29 (3) (1958) 459

[5] Kondorsky E., Physik. Z. Sowjetunion 11 (1937) 597

[6] Kersten M., Grundlagen einer theorie der ferromagnetischen hysterese und der koerzitivkraft (reprinted J.W. Edwaards, Ann. Arbor, 1943).- See also Chikazumi S., Physics of Magnetism (Ed. John Wiley N.Y. 1964) p.p. 261-302

[7] Néel L., Cahiers de Phys. 12 (1942) 1-20

[8] Lord Rayleigh, Phil. Mag., 23 (1887) 225-245

[9] Pry R.H., Beans C.P., J. Appl. Phys., 29 (1958) 532-533

[10] Bertotti G., J. Mag. Mag. Mat., 54-57 (1986) 1556-1560

[11] Williams H.J., Shockley W., Kittel G., Phys. Rev., 80 (1950) 1090-1094

[12] Jiles D.C., Atherton D.L., J. Appl. Phys., 55 (6) (1984) 2115-2120

[13] Jiles D.C., Atherton D.L., IEEE Trans. on Magn., MAG.19 (5) (1983) 2183-2185

[14] Knowles J.E., Proc. of Phys. Soc., LXXV, 6 (19.59) 885-897

[15] Dionne G.F., Weiss J.A., Allen G.A., J. Appl. Phys., 61(8) (1987) 3862-3864

[16] Preisah F., Phys. Z., 94 (1935) 277

[17] Atherton D.L., Spunar B., Spunar J.A., IEEE Trans. on Magn., MAG.23(3) (1987) 1856-1865

[18] Mayergoyz I.D., J. Appl. Phys., 69(8) (1991) 4602-4604

[19] Mayergoyz I.D., Friedman G., J. Appl. Phys., 61(8) (1987) 4022-4024

[20] Del Vecchio R.M., IEEE Trans. on Magn., MAG. 16(5) (1980) 809-811

[21] Middelton F.H., J. Appl. Phys., 32(3) (1961) 2513-2525

[22] Curland N., Speliotis D.E., IEEE Trans. on Magn., MAG. 6(3) (1970) 640-646

[23] El. Sherbiny M.K., IEEE Trans. on Magn., MAG. 9 (1) (1973) 60-61

[24] Saito Y., Hayano S., Nakamura H., Kishino Y., Tsuya N., J. Mag. Mag. Mat., 54-57 (1986) 1613-1614

[25] Rivas J., Zamarro J.M., Martin E., Pereira C., IEEE Trans. on Magn., MAG. 17(4) (1981) 1498-1502

[26] Roshen W., IEEE Trans. on Magn., 27(6) (1991) 4407-4415

[27] Bouc R., Acustica, 24 (1971) 16-25

[28] Hodgton M.L., IEEE Trans, on Magn., 24(1) (1988) 218-221

[29] Wiesen K., Charap S.H., J. Appl. Phys., 61(8) (1987) 4019-4021

[30] Mayergoyz I.D., J. Appl. Phys., 63(8) (1988) 2995-3000

[31] Oti J., Della Torre E., J. Appl. Phys., 67(9) (1990) 5364-5366

[32] Friedman G., J. Appl. Phys., 69(8) (1991) 4832-4834

[33] Globus A., Duplex P., IEEE Trans. on Magn., 2 (1966) 441-445

[34] Perduijn D., Peloschek H.P., Proc. Beit. Ceram. Soc., 10 (1968) 263-273

[35] Globus A., (Thesis - Paris 1963)

[36] Globus A., J. de Phys., 38 (Suppl. C1) (1977) C1-1 -C1-15

[37] Roess E., Hanke I., Moser E., Z. Angew. Phys., 17 (1964) 59

[38] Beer A., Schwarz J., IEEE Trans. on Magn., 2 (1966) 470

[39] Johnson M.T., Visser E.G., IEEE Trans. on Magn., 26(5) (1990) 1987-1989

[40] Gieraltowski J., J. Mag. Mag. Mat., 81 (1989) 103-106

[41] Degauque J., Astie B., Poerteseil J.L., Vergne R., J. Mag. Mag. Mat., 26 (1982) 261-263

[42] Mahnoud S.A., Semary M.A., Mohsen F., Phys. Stat. Sol. (a) 45 (1978) K5-k7

[43] Van der Zaag P.J., Noordermeer A., Ruigrak J.J.M., Por P.T., Rekveldt M.Th., Donnet D.M., Chapman J.N., Proc. of ICF6 (Ferrites- Japan Soc. of Powd. and Powd. metall. Tokyo 1992) pp.819

[44] Visser E.G., Johnson M.T., Van der Zaag P.J., Proc. of ICF6 (see above)pp. 807

[45] Liu C.S., Wu J.M., Nan Lin I., Chen J.C., J. Appl. Phys., 79(8) (1996) 5432-5434

[46] Le Floc'h M., Loaec J., Pascard H., Globus A., IEEE Trans. on Magn., MAG-17(6) (1981) 3129-3134

[47] Konn A.M., Le Floc'h M., Globus A., IEEE Trans. on Magn., MAG-22(5) (1986) 644-646

[48] Le Floc'h M., J. Appl. Phys., 66(3) (1989) 1279-1284

[49] Le Floc'h M., IEEE Trans. on Magn., 25(5) (1989) 4283-4286

[50] Le Floc'h M., J. Appl. Phys., 67(1) (1990) 405-409

[51] Le Floc'h M., Konn A.M., Pascard H., IEEE Trans. on Magn., 26(5) (1990) 2238-2240

[52] Globus A., Cagan V., Le Floc'h M., Pascard H., Loaec J., Proc. of ICF3 (Ferrites-Japan Ceram. Soc., Tokyo-1980)pp.272-275

[53] Le Floc'h M., Konn A.M., Pascard H., Proc. of ICF6 (1992 - see above)pp.796-799

[54] Guyot M., Globus A., J. de Phys., 38 (Suppl. C1) (1977) C1-157 - C1-162 - See also : Guyot M., (Thesis -Paris -1975)

[55] Liorzou Fr., Berthou-Pichavant K., Le Floc'h M., ICF7 (Bordeaux-France-1996) 